This item was submitted to Loughborough's Research Repository by the author.

Items in Figshare are protected by copyright, with all rights reserved, unless otherwise indicated.

\title{
Human factors in the design of medical devices - approaches to meeting international standards in the European Union and USA
}

PLEASE CITE THE PUBLISHED VERSION

http://dx.doi.org/10.1016/j.apergo.2016.08.034

\section{PUBLISHER}

(C) Elsevier

VERSION

AM (Accepted Manuscript)

\section{PUBLISHER STATEMENT}

This work is made available according to the conditions of the Creative Commons Attribution-NonCommercialNoDerivatives 4.0 International (CC BY-NC-ND 4.0) licence. Full details of this licence are available at: https://creativecommons.org/licenses/by-nc-nd/4.0/

\section{LICENCE}

CC BY-NC-ND 4.0

\section{REPOSITORY RECORD}

Privitera, Mary B., Mark A. Evans, and Darren J. Southee. 2019. "Human Factors in the Design of Medical Devices - Approaches to Meeting International Standards in the European Union and USA". figshare. https://hdl.handle.net/2134/22726. 


\title{
Human factors in the design of medical devices - Approaches to meeting international standards in the European Union and USA
}

\author{
Mary Beth Privitera* corresponding author \\ University of Cincinnati \\ Cincinnati, Ohio 45221 \\ United States \\ privitmb@uc.edu \\ +15135580647 \\ Mark Evans \\ Loughborough University \\ Loughborough, UK \\ M.A.Evans@lboro.ac.uk \\ +44 (0)1509 222656 \\ Darren Southee \\ Loughborough University \\ Loughborough, UK \\ D.J.Southee@lboro.ac.uk \\ +44 (0)1509 222662
}

\begin{abstract}
This paper focuses on the challenges of meeting agency requirements as it pertains to the application of human factors in the medical device development process. Individual case studies of the design and development process for 18 medical device manufacturers located in the US and EU were analyzed and compared using a multiple case study design. The results indicate that there are four main challenges in implementing international standards. These include a lack of direct access to users for the purposes of device development; a lack of understanding by users with regards to the impact of their feedback on the development process; contract formalities limiting user exchanges; and the perceived attitudes of clinical users which impact participation in human factors studies. The barriers presented in this research have the potential to be resolved but only with greater commitment by both medical device users and developers.
\end{abstract}

Keywords: medical device, user-centered design, and regulatory requirements

\section{Introduction}

The design of medical devices starts with a need and a description of the concept or problem (Ogrodnick 2013). In order for a new device to be used within the clinical environment, certain development procedures must be undertaken, such as a regulatory 
plan and design optimization through verification/validation (Whitmore 2004; Zenios et al. 2010; Ogrodnik 2013). Furthermore, the integration of human factors in medical device design process is required to reduce risk and improve patient safety (FDA 2011; MHRA 2016). Additionally, design Control is a fundamental requirement to meet regulatory approval for international standards (FDA CDRH, 1997; Ogrodnick 2013). It documents the history of development and ensures that the origins of any decision made during the development process are traceable. In order to improve the ability of designers and auditors to ascertain the safety and efficacy of a product, the use of design controls has been adopted in order to specify the appropriate method for device review at several key stages (Gilman et al. 2009). The framework of this model meets United States (US) Federal Regulation 820.30 for Design Control (Justiniano and Gopalaswamy 2003; Gilman et al. 2009; Panescu 2009). For example, in determining user needs, ethnography and/or contextual inquiry is undertaken in order to write formative usability objectives that meet industry standards for the Association for the Advancement of Medical Instrumentation (AAMI) (AAMI, 2009)/American National Standards Institute (ANSI) Human Engineering Standard 75 and International Electrotechnical Commission (IEC) (IEC 62366: 2007/(R) 2013). Wilcox (2012) explains the use of ethnographic research in medical device development in order to learn what actually takes place, as opposed to what people say takes place and then using that information to create devices that reduce error and improve productivity. This paper focuses on the challenges of meeting these agency requirements as it pertains to the application of human factors in the medical device development process in both the US and the European Union (EU). This furthers the research of Vicent et al (2014), wherein barriers, such as communication breakdowns between users and design teams hindered integrating human factors in medical device design (Vincent et al. 2014). For reference, the human factors standards applicable to medical device development are as follows:

- IEC 62366-1:2015, Medical devices - Application of usability engineering to medical devices, published by the International Electrotechnical Commission.

- IEC/TIR 62366-2:2016, Medical devices - Part 2: Guidance on the application of usability engineering to medical devices, published by the International Electrotechnical Commission. (Note: this standard was not published at the time of this study)

- AAMI HE 75:2009, Human Factors Engineering - Design of Medical Devices, Section 9 - Usability Testing, published by the Association for the Advancement of Medical Instrumentation.

- Medical Device Safety - Integrating Human Factors Engineering into Risk Management, June 18, 2000, available on the U.S. Food and Drug Administration's website.

- FDA's guidance document Applying Human Factors and Usability Engineering to Medical Devices, which addresses usability testing, issued on February 3, 2016, available on the U.S. Food and Drug Administration's website. (Note: this guidance document was in draft during the time of this study)

- ISO 14971:2007, Medical devices - Application of risk management to medical devices, published by the International Organization for Standardization.

In the UK, the Competent Authority is Medicines \& Healthcare products Regulatory Agency (MHRA). The MRHA enforce the following regulations (MHRA 2016): 
- The Medical Devices Regulations 2002 - SI 2002/618 (consolidated legislation)

- $\quad$ The Medical Devices (Amendment) Regulations 2003 - SI 2003/1697

- The Medical Devices (Amendment) Regulations 2007 - SI 2007/400

- The Medical Devices (Amendment) Regulations 2008 - SI 2008/2936 (transposes Directive 2007/47/EC into UK law (came into force March 2010))

In 2016, The MHRA published draft guidance titled, "Human Factors and Usability Engineering - Guidance for Medical Devices Including Drug-device Combination Products" (MHRA 2016). This document was not available at the time of the study; however, it provides an overview of the regulatory framework, the standards, process of usability engineering, simulation and post-market surveillance for the EU. As evidenced by these international requirements, the application of human factors is critical to the device approval process.

For clarity, the definition of who a user is in terms of a medical device can vary. It can be a clinical provider, the patient, a parent or caregiver. For example, Money et al. (2011) identify the user as any healthcare worker who may be required, either regularly or occasionally, to locate, examine or access blood vessels of patients or to assist with these tasks. De Ana notes that careful analysis of all stakeholders is required to determine precisely who the user is for any device in development as it may be that through the use of the device the user may change from the provider to the patient or vice versa (De Ana et al. 2013).

Kaye (AAMI 2013) comments on the fundamental challenge in medical device design as being, "...... the ability to identify and understand, particularly during design, possible use problems and the potential for harm associated with the use of medical devices. Additionally, many system-use problems were context-specific, subtle, complex, and hard to identify. However, use related problems can be detected via formative testing and evaluation by users " (AAMI 2013 p.9). This can be seen as being indicative of the importance of early and direct user involvement before medical device development. Observational research, such as contextual enquiry and usability testing, formalize user involvement in the design process at specific points, with all users being sensitive to device usability along with safety and efficacy (Wiklund and Wilcox 2005). However, it is not possible to have every element of the design perfected to all user expectations in addition to 
meeting the needs of safety, efficacy, and technical feasibility. Fairbanks and Wears (2008) sum up the perception of users towards industry stating that device manufacturers should ".... presume there to be a design problem rather than a user problem and work from that starting point to find avenues for improvement...we cannot be satisfied with weak solutions that provide the illusion of action but will accomplish little or nothing, such as a new policy, exhortation, and training." (Fairbanks and Wears, 2008 p. 520)

In a study by Money et al. (2011), in-depth interviews were conducted with representatives from 11 medical device manufacturers. They asked the manufacturers to identify whom they believe the intended users were; what role they had in the process; and what value (if, any) did they believe the users added. They used thematic analysis to review transcripts and found there were perceived barriers to specific user groups in obtaining ethical approval, the speed at which such activity may be carried out and belief that there was no need to seek user input given the 'all-knowing' nature of senior healthcare staff and clinical champions (ibid). Additionally, only one manufacturer claimed to regularly use formal user-centred design methods within the development process. Hence, the only evidence of engagement with users was the formal methods that were mandatory and dictated to manufacturers by standards and purchasing agencies. Money et al (2011) suggests the focus of IEC 62366 that device manufacturers should:

- "Research to better understand the requirements of manufacturers, in terms what was required from human factors engineering methods in order to make their use more feasible and accessible in practice.

- Provision of training on the use and benefits of employing formal human factors engineering methods at every stage of the medical device development process.

- Healthcare providers should implement formal processes to ensure better communication and synergy between those making purchasing decisions and the actual users of the devices.

- Provisions should be made within the ethical approval process that enables medical device manufacturers to engage more easily with users with minimal levels of bureaucracy while also ensuring that all research was conducted in an ethical manner that protects healthcare staff and patients" ( Money et al.. 2011 p.11) 
As a result of agency mandates and standards such as IEC 62366, users (customers) are most often involved in need determination, usability assessment and the development of surgical technique (Ogrodnick 2013). Usability is considered as being important although not enough on its own to guarantee product success. Usability techniques can be used to improve a given situation but they do not reveal if a given situation is better or more enjoyable (Battarbee and Koshinen 2005).

A realization that human error in operating a device can be a major cause of patient death or injury (Cafazzo \& St-Cyr 2012) identifies a need for collaboration throughout the design process rather than at formal mandated intervals. As collaborative design techniques become more widely adopted, the role of the user has started to change. In consumer product design, this calls for participation of users within the design process that involves users as co-creators (Sanders 2002). It changes the perception of the role of users within the design process to nurture collective creativity, where users are active, competent participants (Binder et al. 2008).

Lin and Vicente (2001) comments that manufacturers have the capacity to enhance patient safety by putting a greater emphasis on the human factors engineering process in the design of devices. This position is expanded by suggesting that government medical regulators may be able to enhance patient safety by putting an emphasis on human factors engineering design criteria when undertaking final product approval and regulatory decisions (ibid). Specifically, the incorporation of human factors engineering methods within the medical device design and development process offer benefits that facilitate safer and more usable devices that are better suited to user needs (Money et al. 2011). These methods include focus groups, interviews, usability testing and heuristic evaluation once a tangible device has been developed. As devices are released for use, hospital risk managers can enhance patient safety by adopting human factors engineering criteria during the evaluation of a new technology (Lin et al. 2001).

Government mandates are one of the most powerful influences on medical device development. They impose and recognize the rules or standards necessary to 
market a device and have required post-market surveillance that is intended to identify issues as they occur. Like the US ANSI/AAMI standards body, device manufacturers undertake the MRS on a voluntary basis. The National Health Services (NHS) in the UK uses the platform of standards in order to identify suppliers whose products have a measure of quality assurance and meet the requirements of the quality system standard (Crisp 1996). Both the USA and the EU require the demonstration of a human factors engineering process throughout design/development and upon submission of a device dossier being assessed for device approval to market (Martin and Barnett 2012). In all instances, the regulations and procedures required by the agencies describe what the documentation should contain but do not prescribe how the design/development process should actually be undertaken (Ogrodnick 2013).

\section{Methods}

Case studies are effective in the assessment of user engagement and in capturing design and development practices (Elf et al. 2007; Grocott et al. 2007; Taylor et al. 2007; Medina et al. 2012; Yin 2013). Unlike the analysis of an individual case, this study compares practices from multiple cases that were selected based on the size of the company, device specialty, user group and use of industrial design. Yin (2013) identifies case study research as being appropriate when the main research questions are 'how' or 'why' and where the researcher has little control over behavioral events. Yin (2009) further describes case studies as coping with technically distinctive situations with more variables of interest rather than data points and the reliance on multiple sources of evidence. As such, multiple case designs were considered as being more compelling, making the overall study more robust when replication logic was followed.

Data analysis of case studies consists of using a standard set of variables with openness to new variables in order to form a foundation. Once each case is understood, case level displays can be 'stacked' in a meta-matrix, thereby further condensing and permitting systematic comparison (Miles et al. 2013). For this study, a graphic data display was used to develop a meta-matrix enabling the cross-referencing of variables.

Figure 1 represents the template used to visualise each participating manufacturer. The graphic represents the specific requirements for each organisation in one graphic element and was used to enable analysis based on each specific requirement. All manufacturers selected to participate in this study produced one or more of the following: Class II devices used in general hospital (GEN), critical care (CRIT), drug delivery (DELIV), surgery (SURG) or catheter based interventions (CATH). The devices manufactured by participants included users who were physicians (DR), patients (PT), 
technicians (TECH), nurses (NRS), and care providers (CARE) such as home healthcare providers. Additionally, they ranged in size from small < 50 (SM), medium 51-149 (MED) and large >150 (LG) and additional consideration was given to in-house industrial design (ID) or the lack thereof (NO ID). A large number, represented by the $\mathrm{X}$ in Figure 1, provided a unique identifier for each company.

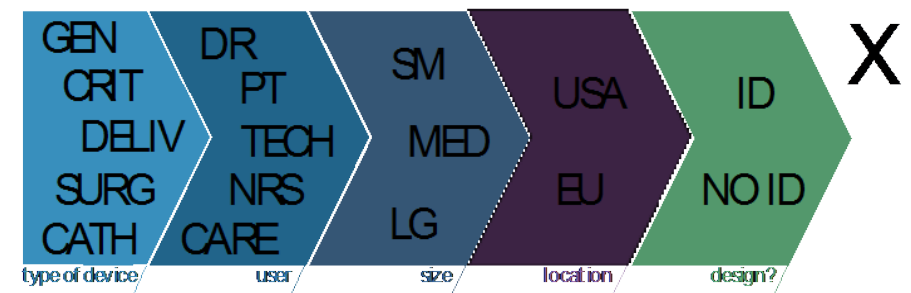

Figure 1. Visual description used for each manufacturer

A total of 18 Individual medical device manufacturer cases were developed using a combination of techniques. This study is limited to device manufacturers as they are the responsible party for executing human factors studies. These included exploring the company background to determine the number of employees, product portfolio and regulatory classification of the devices. In addition, the profile of the participant was reviewed via social media on professional web sites such as LinkedIn. Criteria for case study development included the type of device, the user, the size of company, the location and use of industrial design. Semi-structured interviews were conducted, with 13 being undertaken at the participant's place of work and five by Skype. The interviews were conducted using an interview guide approach (Bauer 1996; Kvale and Brinkmann 2009) and lasted approximately one hour. In order to assess each manufacturers opinions regarding user centered design and human factors, questions were directed at opening conversations on five distinct areas of: development process or approach to design; challenges of engaging users; methods; frequency of engaging users; and the influence of industrial design in regards to the process. The interview questions consisted of the following:

1. What is the development process/theoretical approach to design? (Probe for user-centred design, participatory design, collaborative design, evidence of human factors process)

2. What are the challenges in involving users within your design process? (Probe for specific tools, activities, requirements such as IEC 62366 that drive user involvement) 
3. How often/where does the engineering/design team engage with users? (Probe for process of engagement, human factors study execution)

4. Do you have industrial design (ID) within your organization? (Probe for the hiring of consultants: ID or human factors and responsibilities)

These areas were selected as being the most important in order to meet the research objectives as all questions revolve around user input into the design process and all are required to meet agency requirements. At the conclusion of the interview, specific artifacts, such as corporate guidance on design approach/design process training material or interesting artifacts discovered in the interview, were requested in order to provide further understanding and enable data analysis using a meta matrix display.

Figure 2 illustrates the collective background on each manufacturer. This figure describes the range in the types of users, with 3 companies developing two different types of devices; 9 companies with multiple types of users; and including the size, location and presence of industrial design illustrated for all companies. 
m

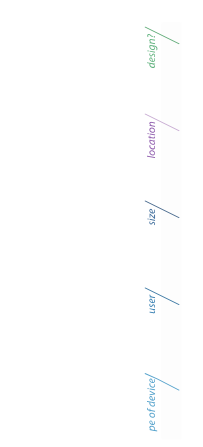

$\Lambda$
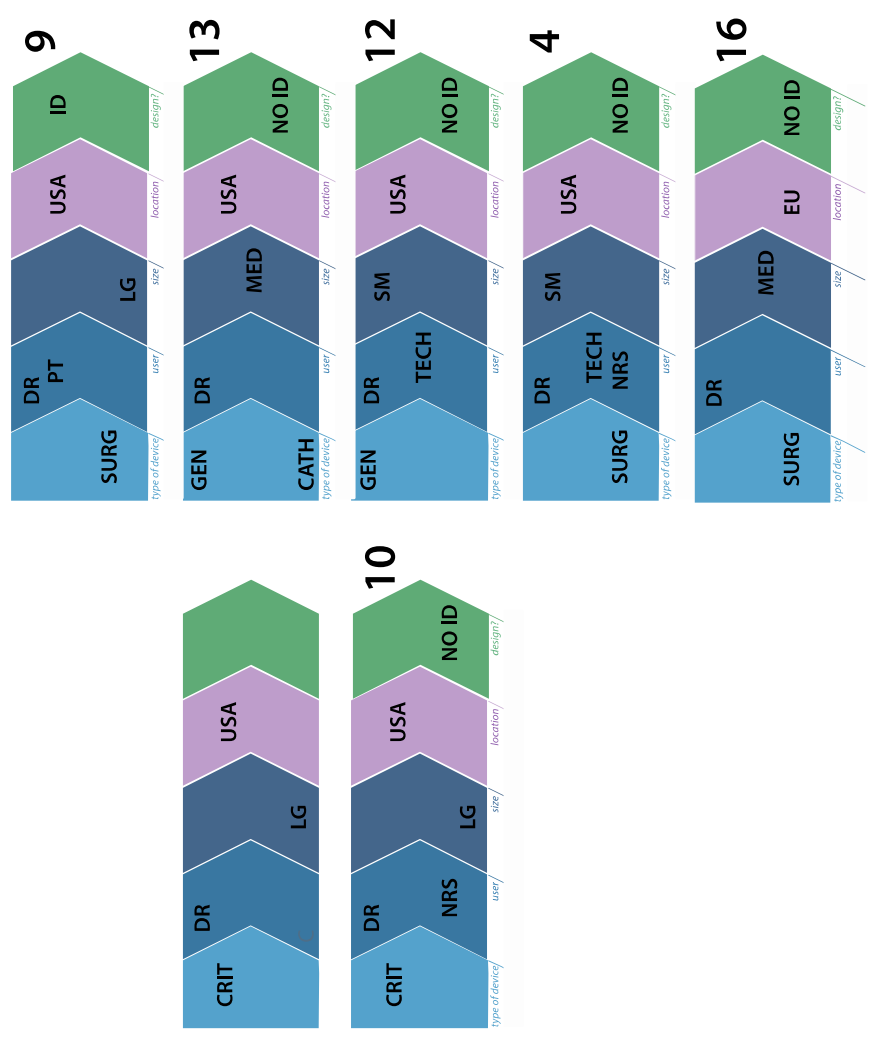

Figure 2. Graphic details of participating manufacturers 
With permission from the interviewees, all interviews were recorded using a Livescribe pen for reference and analysis that complied with ethical procedures of the University of Cincinnati. Due to manufacturer confidentiality, no images were taken.

Medical Device Industry (MDI) manufacturers included interviewees from 13 USA, three EU and two contract manufacturers. Examples of the types of the manufactured devices included laparoscopic surgical devices, orthopedic implants, assistive surgical technologies, endovascular devices, neurovascular devices, critical care devices, cardiac assistive technologies, neurologic diagnostic devices and general hospital equipment such as patient administered drug delivery devices. Selected manufacturers included major medical device developers from each target area. Table 1 provides a summary of the size and location of the participating companies.

Table 1. Medical device manufacturer participant breakdown

\begin{tabular}{|l|l|l|l|l|}
\hline Company & Small $<50$ & Medium 51-150 & Large $>150$ & Total \\
\hline US Device Manufacturer & 3 & 2 & 8 & 13 \\
\hline EU Device Manufacturer & 1 & 1 & 1 & 3 \\
\hline US Contract Manufacturer & & 2 & & 2 \\
\hline
\end{tabular}

All interviewees held leadership positions within their organization, therefore responsible for the design process and had an average of 12 years of experience. Table 2 describes the participating disciplines and leadership levels. Interviewees were selected based on their leadership within the organization.

Table 2. Interviewees by discipline

\begin{tabular}{|l|l|l|l|l|}
\hline Discipline & Manager & Director & Vice President & Chief Technical Officer \\
\hline Engineering & 2 & 4 & 2 & 3 \\
\hline Industrial Design & 3 & 2 & & 1 \\
\hline Human Factors & & & 1 & \\
\hline
\end{tabular}


Qualitative samples tend to be purposive rather than random (Miles et al. 2013). As such, the selected manufacturers had to meet the following requirements (Table 3):

- Type of device - Must manufacture at least one Class II device, which is a regulatory device classification and used in/for the catheter lab (CATH), critical care (CRIT), drug deliver (DELIV), surgery (SURG) or general hospital (GEN)

- User - Any user group was welcome, however, at least one use location must be in a hospital in order to bound the collected data. User groups included physicians (DR), nurses (NRS), patients (PT), caregiver (CARE) and technologists (TECH).

- Size of company - A mix of large (LG) medium (MED) or small (SM) was targeted

- Location - Must be located in either the USA or EU

- Industrial Design (ID) - Must be familiar with the use and value of industrial design

Table 3 Participant summary indicating the device type, user group, size, location and presence of ID

\begin{tabular}{|c|c|c|c|c|c|c|c|c|c|c|c|c|c|c|c|c|c|c|}
\hline Company \# & 1 & 2 & 3 & 4 & 5 & 6 & 7 & 8 & 9 & 10 & 11 & 12 & 13 & 14 & 15 & 16 & 17 & 18 \\
\hline $\begin{array}{l}\text { Device Type } \\
\text { (CATH, CRIT, } \\
\text { DELIV, SURG, } \\
\text { GEN) }\end{array}$ & CATH & CATH & SURG & SUAG & SURG & GEN & $\begin{array}{l}\text { GEN, } \\
\text { SURG }\end{array}$ & GEN & SURG & CRIT & CRIT & GEN & $\begin{array}{l}\text { GEN, } \\
\text { CATH }\end{array}$ & SUAG & DELUV & SURG & SURG & $\begin{array}{l}\text { GEN, } \\
\text { SURG }\end{array}$ \\
\hline $\begin{array}{l}\text { User } \\
\text { (DR, NRS, PT, } \\
\text { TECH, CARE) }\end{array}$ & DR & DR & $\mathrm{DR}$ & $\begin{array}{l}\text { DR, } \\
\text { TECH, } \\
\text { NRS }\end{array}$ & $\mathrm{DR}$ & $\begin{array}{l}\text { DR, } \\
\text { PT, } \\
\text { TECH, } \\
\text { NRS, } \\
\text { CARE }\end{array}$ & \begin{tabular}{|l|} 
DR, \\
PT, \\
NRS, \\
CARE
\end{tabular} & DR & $\begin{array}{l}\text { DR, } \\
\text { PT }\end{array}$ & $\begin{array}{l}\text { DR, } \\
\text { NRS }\end{array}$ & $\begin{array}{l}\text { DR, } \\
\text { NRS }\end{array}$ & $\begin{array}{l}\text { DR, } \\
\text { TECH }\end{array}$ & $\mathrm{DR}$ & DR & PT & DR & $\begin{array}{l}\text { DR, } \\
\text { TECH }\end{array}$ & $\begin{array}{l}\text { DR, } \\
\text { PT }\end{array}$ \\
\hline $\begin{array}{l}\text { Size } \\
\text { (SM, MED, LG) }\end{array}$ & LG & MED & LG & SM & LG & LG & MED & SM & LG & LG & LG & SM & MED & LG & LG & MED & SM & MED \\
\hline $\begin{array}{l}\text { Location } \\
\text { (USA, EU) }\end{array}$ & USA & USA & USA & USA & USA & USA & USA & USA & USA & USA & USA & USA & USA & USA & EU & EU & EU & USA \\
\hline ID (YES, NO) & NO & NO & NO & NO & NO & YES & NO & NO & NO & NO & NO & NO & NO & YES & NO & NO & YES & YES \\
\hline
\end{tabular}

Cross-case synthesis can be conducted by treating each individual case as a separate study and then aggregating findings across a series of studies (Yin 2013). As such, "analysis is likely to be easier and the findings likely to be more robust than having only a single case" (Yin, 2013 p. 164). Cross-case data requires comparison via common codes, common displays of data segments and reporting formats for each case (Miles et al. 2013). All codes, displays and reporting formats are datacondensing for the purposes of distilling data sets into "workable, intellectual coherent units" (Miles et al. 2013 p. 136). 
To identify overall similarities and differences between multiple cases, a card sorting technique was employed as these are widely used in knowledge acquisition (Rugg and McGeorge 2005). Card sorting also supports the goals of organizing information into a usable taxonomy, with consideration to differences between groups, initially as a whole and then as individuals (Bussolon 2009). For this analysis, each card contained uniform information regarding the size, location, user and type of device, in-house industrial design, profession of interviewee and brief answers to the main study questions. An individual card was written for each company and then organized based on responses. This comparison analysis enabled a broad overview of each topic. Figure 3 highlights a card sorting analysis for design approach.

\section{Engineering}

Figure 3. Card Sorting Example

Further, a data display is an organized visual containing a compressed assembly of information that allows conclusion drawing and action (Miles et al. 2013). Further mapping and data visualisation were used to determine patterns across case studies. A matrix display refers to a display chart or table including the codes in order to organise a vast array of condensed material into an at-a glance format for reflection, verification and conclusion drawing (Miles et al. 2014). In order to facilitate further data analysis, data visualisation charts were developed using individual case images 
based on Figure 2. An example chart can be seen in Figure 4. The vertical axis communicates the total number of participants reporting a finding. The horizontal axis represents various topics identified from the data. By using individual company graphics that include the specific requirements, further analysis based on company attributes was enabled. From this visualisation, the data was analysed in both the vertical and horizontal axes. These charts were used for noting patterns, explanations, causal flows and propositions in order to draw conclusions.

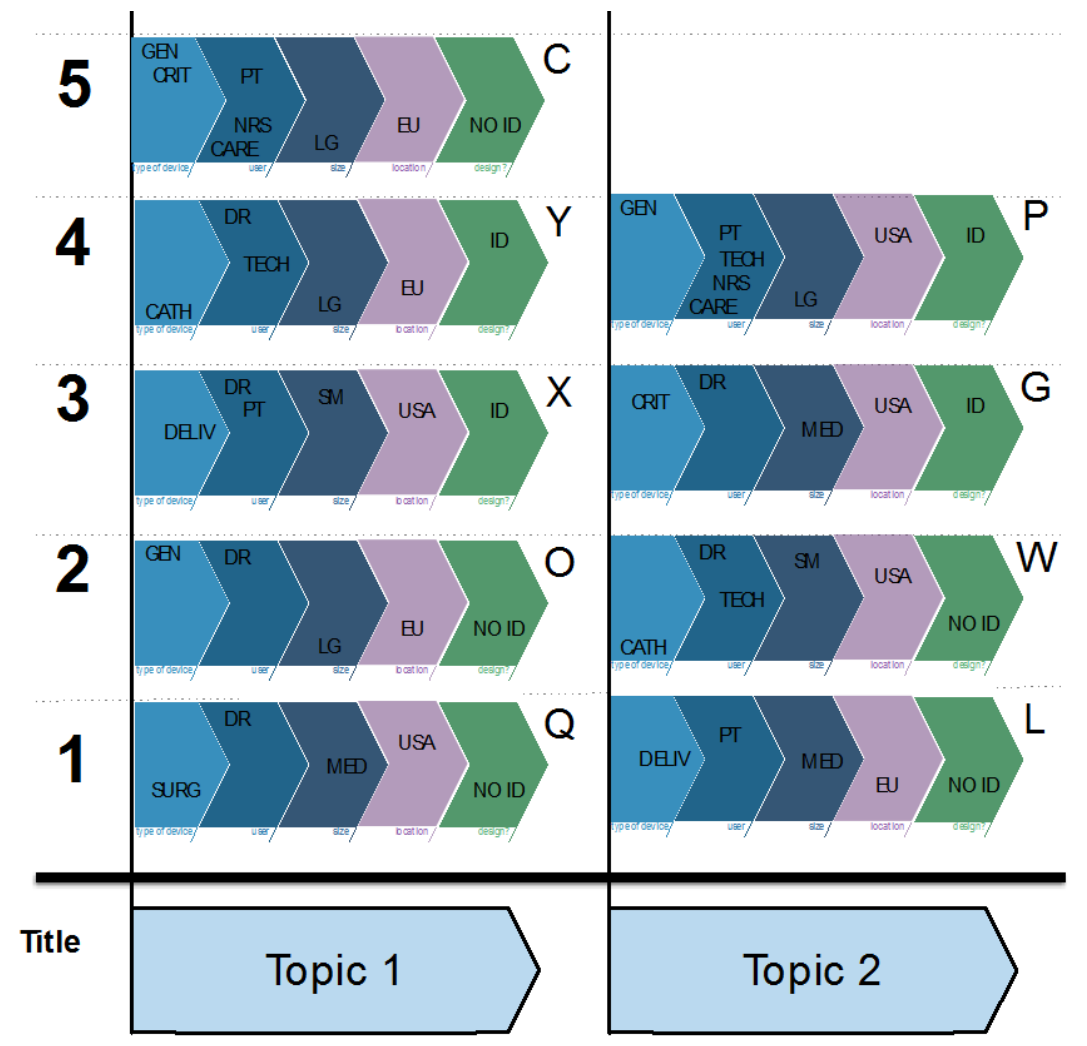

Figure 4. Data visualization example

\section{Results}

Each of the human factors standards required for agency review involves collaborative exchanges with users. As such, the results are presented with regard to the means of user involvement; activities used promoting collaborative exchanges; and the challenges with user involvement. 


\subsection{Means of user involvement}

All manufacturers involved users in the design process. Table 4 indicates the type of user involvement as described by interviewees. A total of 13 companies had users involved through fee-based consulting agreements with medical device professionals immersing themselves and observing the clinical environment. These manufacturers had users involved in formal device studies, noting that this was an agency requirement prior to approval to market a device.

Table 4. Typical user involvement in device development

\begin{tabular}{|l|l|l|}
\hline $\mathrm{N}=$ & User Involvement & Activities or requirements involving users \\
\hline 13 & Consultancy fee & Only interact with users who are dedicated consultants \\
\hline 13 & $\begin{array}{l}\text { Immersion \& } \\
\text { observation }\end{array}$ & $\begin{array}{l}\text { Activities by the product development team members to } \\
\text { more fully understand context of use }\end{array}$ \\
\hline 12 & $\begin{array}{l}\text { Formal device } \\
\text { assessment }\end{array}$ & Requirement for user involvement by regulating agencies \\
\hline 7 & Advisory group & $\begin{array}{l}\text { Group of users selected to provide guidance in device } \\
\text { development }\end{array}$ \\
\hline 7 & $\begin{array}{l}\text { Informal } \\
\text { interactions }\end{array}$ & $\begin{array}{l}\text { Activities not formally related to device development but } \\
\text { use to build working relationship }\end{array}$ \\
\hline 6 & $\begin{array}{l}\text { Key opinion } \\
\text { leaders (KOLs) }\end{array}$ & $\begin{array}{l}\text { Selected users who have influence on clinical practice, } \\
\text { typically through publication and/or procedure } \\
\text { development }\end{array}$ \\
\hline
\end{tabular}

A total of 7 companies had clinical advisory groups or reported informal interactions with users. Three of these Companies $(16,13$, and 3 ) utilized both. Uniquely, Company 3 involved users as advisory group members for informal interactions and as key opinion leaders. Likewise, Companies 14 and 2 reported advisory group and key opinion leaders. In these instances, there were individual contracts with physicians as they served in both capacities. 
Users frequently received a fee through direct consulting, honoraria or donation and, in contextual enquiry studies, this extended to the hospital or institute where they practiced. The act of simply observing care or involving users resulted in financial expectations, especially for those practicing in larger hospitals. In addition, larger organizations, such as Company 15, often used a third party recruiter to identify study participants, thereby further increasing the cost. A mid-size or start-up device company (Company 17), although hesitant to compensate, they would only do so if resources were available and it was absolutely necessary. In these instances, greater emphasis was placed on relationships with individuals. All companies used formal non-disclosure and non-compete agreements as routine practice when collaborating with users.

Front-end user studies, such as contextual enquiry, were conducted by two of the large manufacturers (Companies 3 and 9). These were described as time intensive studies, tying use behaviors within the clinical workspace to specific device performance outcomes. Industry participants described that the number of users or stakeholders involved in these studies had increased to include those who were considered ancillary users (those who prepared the device for use) and that these stakeholders had a voice in the purchase decision. User participation often ceased (briefly) between a contextual inquiry study/unmet needs investigations and the development of intellectual property (Companies 3,6,7,10, 11, 14).

Participants conducted formal device assessments, wherein users were brought to an external facility such as a hotel, to provide feedback on a device as required by IEC 62366 (Companies 2, 3, 6, 11, 14, 16). If users were patients themselves or the device was intended for home use, the evaluations were conducted through collaboration with clinical personnel in order to identify appropriate patients, with the patients themselves being asked to use the device under direct observation following a summative usability assessment protocol. This was documented and placed in a human factors dossier for agency review. These validation protocols followed a scripted research protocol.

One large manufacturer had put together clinical advisory boards consisting of KOL's, with formal consulting agreements (Company 14). This advisory board was active in 
assisting in directing the product development strategy as well as assisting in the development of key attributes that made the device more acceptable to those making purchase decisions. All members of the board received a fee and were required to maintain a design notebook to record intellectual property. They were made up of clinical members who were influential in their community and served to assist the design team. The reviews by the advisory group were formal planned activities. The opinions of the study participants were that these boards were "not reflective of the mass market, rather they were only the top 5\% of the world" (Company 3). It was noted that feedback from the advisory board was different than feedback gathered from formal human factors studies. Additionally, this advisory group was known to "have many ideas however get upset and take it personally when negative feedback (regarding a concept) was delivered" (Company 1). This indicates the formal studies were less bias.

Select users were involved in the development process through formal device evaluations or as part of an advisory group, often being made up from key opinion leaders (KOL's). Those involved were generally selected based on their personality or achievements in research and potential influence over other practitioners. One small manufacturer (Company 12) commented on collaborative relationships in which the lead clinical support was a member of the leadership team although they no longer practiced medicine. As a result, further collaborations with practicing clinicians were routinely sought for feedback.

Informally, the device industry was routinely exposed to clinical practice. Unlike contextual inquiry studies, with a planned immersion and observation activities, this was often ad hoc and conducted alongside 'voice of customer' marketing research. There was a realization that just asking questions was not enough information to drive design criteria as there was a need to explore opportunities more fully and gain a deeper understanding of practice. This was described as "leading to better questions and more defined user input" (Company 3). During these interactions, notes were occasionally taken although there was a tendency by industry personnel to be present in the observation and then reflect on the visit when completed. 


\subsection{Activities promoting collaborative exchanges}

Device companies enabled the inclusion of users in the device development process through specific activities. Following data reduction, four types of activities were identified (see Table 5). A total of 17 companies engaged users in specific device studies or reviews, with 13 reporting the involvement of users in workshops or device related labs. Eight reported the involvement of users in human factors evaluation, with five companies having in-house industrial design teams. Four companies without industrial design capability were engaged in medical device design related conversations at scientific conferences. Additionally, all of these companies engaged with physicians as users, with these including both of the catheter device manufacturers.

Table 5. Activities promoting user involvement

\begin{tabular}{|l|l|l|}
\hline $\mathrm{N}=$ & Activities promoting user involvement & $\begin{array}{l}\text { Specific interactions of the user with } \\
\text { medical device development teams }\end{array}$ \\
\hline 17 & $\begin{array}{l}\text { Device Study or Review: Informal } \\
\text { discussion }\end{array}$ & $\begin{array}{l}\text { Conducting a discussion on design } \\
\text { concepts (in office or lab) }\end{array}$ \\
\hline 13 & Workshops and labs & $\begin{array}{l}\text { Conducting specific design events to } \\
\text { promote user engagement }\end{array}$ \\
\hline 8 & $\begin{array}{l}\text { Human factor evaluations: Formal study } \\
\text { meeting IEC 62366-1 requirements }\end{array}$ & $\begin{array}{l}\text { Design attributes analyzed using } \\
\text { formal study }\end{array}$ \\
\hline 4 & Scientific conferences & $\begin{array}{l}\text { Discussion and presentation during } \\
\text { user focused meetings }\end{array}$ \\
\hline
\end{tabular}

Device use was studied through ad hoc observations and contextual enquiry that was conducted before design control, thereby exploiting customer databases held by the manufacturer. A multidisciplinary team was always involved in the study, with one company highlighting "the ability to organize the information into key insights that were actionable by design" (Company 7). The impact of a formalized contextual enquiry program was recognized in one large organization by office conversations based on "what problems were we trying to solve, rather than what color or less important details" (Company 10). Ad hoc observations were also used to assist in 
"learning new techniques, relationship building or to explore for specific project exploration" (Company 2). For these visits, only senior engineering and marketing personnel were typically involved in observations for mid-sized companies. In contrast, large companies only observed care as part of a formal study. A deficiency identified in this approach was "the reliance on individual note taking with no good way to sort relevancy" (Company 2).

Workshops and labs were used to promote collaboration between device developers and users. In these instances, specific time was set aside from clinical care responsibilities for the purpose of exploring a device concept, procedural training for design team members, or to serve as a prompt for device ideation. During labs, the clinical personnel acted as trainers for designers and engineers and walked through important details using demonstrative models such as an animal or cadaver. This was identified as a costly approach but viewed as an important event for start-up organizations. There was typically no formal protocol with regards to the information to be learned although there was a formal protocol submitted to animal protection agencies identifying the purpose and scope of the exploration. This activity was surgical device manufacturers conducted approach. The design and usability information gathered during these session were not recorded in a formal report rather gathered ad hoc and not entered into formal data management systems.

Due to agency mandates, eight manufacturers mentioned human factors and/or usability assessments meeting the requirements of IEC 62366-1. In these instances, a specific usability lab or facility was rented for device review. In others, the use of rapid prototyping with low fidelity functionality was used to review a device concept, with Company 3 commenting: "we were a company full of really smart people, who used to think it's impossible that the user does not think like us, however with user testing, we've learned our assumptions can be completely wrong". One approach by a mid-size company was to produce a concept and then return back to the user to modifications and adjustment in order to meet their needs. In this approach, only one variable was adjusted at a time. The common opinion for device reviews was that "users get overwhelmed by too many choices" (Company 12). As a result, users were provided with only 2 options before being asked to verbally choose a 
preference. Then they were asked to explain the pros/cons of their decision with each iteration of options becoming closer to the final design of the device. In these tests, user preferences generally remained consistent however, the description of context and background as to reasoning was inconsistent thus resulting in challenges when interpreting the data.

In-depth interviews were conducted at scientific meetings as a means of gathering opinion regarding challenges and trends in the market. These were often lead by the company's marketing team and did not acquire rich design information unless there was "someone on the team who knows how to ask the right questions and then translate it into design criteria" (Company 11). There was, typically, no formalized method of synthesizing the data, with the team discussing issues and the making their "best guess" (Company 3). For those not involved in formal contextual inquiry studies, this approach was the alternative and described as "we do free flow conversations with users taking a spark of an idea and letting the tangencies of the conversation flow." In three instances, there was follow-up with users via email for the purpose of new device design rather than to further evaluate specific design concepts. For home health devices, users were provided with diaries with a request to record "how they felt at a particular moment during device use" (Company 15). These diaries were used as interview memory prompts in order for the user to recall and justify their opinion.

\subsection{Challenges with user involvement}

The opinion of device manufacturers included specific reference to positive interactions with users when they were engaged in device development. This was largely attributed to the fact that, in the visits to hospitals or care centers by product development professionals, there was an exchange of information but no selling. While this was seen as being positive, negative attributes arising from collaboration with users were identified. Table 6 describes specific challenges as described by study interviewees. 
Table 6. Industry challenges with user involvement

\begin{tabular}{|l|l|l|}
\hline$N=$ & $\begin{array}{l}\text { Challenges to user } \\
\text { involvement }\end{array}$ & $\begin{array}{l}\text { Hindrances of the development team which prevent } \\
\text { user collaboration }\end{array}$ \\
\hline 11 & $\begin{array}{l}\text { Lack of user knowledge } \\
\text { of product development }\end{array}$ & $\begin{array}{l}\text { Users level of understanding in product } \\
\text { development practices }\end{array}$ \\
\hline 10 & Access & $\begin{array}{l}\text { Making the connections and approvals for a } \\
\text { meeting with users }\end{array}$ \\
\hline 10 & User expectations & $\begin{array}{l}\text { Un-communicated requirements by the user which } \\
\text { negatively impact the relationship or unrealistic } \\
\text { expectations as to what was possible by design }\end{array}$ \\
\hline 7 & Scheduling with users & $\begin{array}{l}\text { Coordinating calendars and variability of clinical } \\
\text { practice }\end{array}$ \\
\hline 7 & Attitude and ego & $\begin{array}{l}\text { Personality influences on collaboration and team } \\
\text { dynamics }\end{array}$ \\
\hline 6 & Conflicting user opinions & Sense making with opposing viewpoints \\
\hline 5 & Cost of involving a user & User compensation for involvement \\
\hline 5 & $\begin{array}{l}\text { Healthcare law } \\
\text { compliance }\end{array}$ & $\begin{array}{l}\text { Legal ramifications of laws resulted in a lack of } \\
\text { desire to be involved with device companies }\end{array}$ \\
\hline 2 & $\begin{array}{l}\text { IRB/ethic board } \\
\text { requirements }\end{array}$ & $\begin{array}{l}\text { Requirements for user reviews such as contextual } \\
\text { inquiry and formal usability studies, viewed as } \\
\text { burdensome for development }\end{array}$ \\
\hline 2 & $\begin{array}{l}\text { User creativity } \\
\text { information }\end{array}$ & $\begin{array}{l}\text { The extent to which a medical device user was able } \\
\text { to envision an alternative clinical approach }\end{array}$ \\
\hline 1 & $\begin{array}{l}\text { Translating what they say } \\
\text { from users by development teams. }\end{array}$ \\
\hline
\end{tabular}

A total of 11 companies reported that the users' lack of understanding of the product development process was a challenge. Of the 11,8 of these companies did not have in-house industrial design and involved all user groups. The fact that users had limited knowledge of the product development process was identified as a significant burden and resulted in unclear communication with developers. In one example, physician innovators with knowledge of the process were eager to have 
their device on the market but did not have an appreciation of the amount of time required for development and testing. Additionally, users could become impatient at the questioning by product development team members as they sought a fundamental understanding of the true clinical need as indicated by Company 13 , "Medical device design teams spend considerable time understanding the clinical problem, target outcomes, and associated risks; whereas users have no idea if the device in question was in what stage in the development process" (Company 13).

Gaining access to clinical care was a challenge for all user types and reported by 10 manufacturers. Members of a development team could not simply schedule an appointment with a user and complete an observation without having to go through a process of preparation and planning. As a general rule, special training and credentials must be submitted to a hospital well ahead of a visit. Simple shadowing, which largely went undetected prior to the introduction of patient privacy laws, were halted by hospital administration if not planned in an appropriate manner. For large companies (Company 14, 10, 6, 5, 3), this almost always involved an additional network and contact with a sales manager or representative and there was an unwritten rule that an engineer was never allowed to have interaction with a user without prior approval and acknowledgement by the sales team. Smaller companies $(\mathrm{N}=4)$ relied on personal relationships and repeated visits within the same institution in order to gain trust. For device evaluations, device companies used a recruiter to avoid interruption with the sales relationships and to maintain blinded studies. In two companies there was a trend in the device industry to only send more senior engineers for specific observations, leaving junior team members to learn about clinical practice through online videos and, in a few instances, specific engineering training labs.

A total of 10 manufacturers reported that users had expectations of rights to a share of the intellectual property (IP). Communicating that IP had already been filed became a balance in the conversation, potentially causing a rift in the collaborative relationship. Companies described an expectation of users to evaluate more developed and robustly designed prototypes. As a result, this could lead to delays in gathering feedback and a desire to demonstrate lower fidelity prototypes for evaluation (Company 14). There was an attitude that users struggled to provide true 
and valuable feedback when presented with relatively simple concept sketches. The challenge for the manufacturer was to measure the right goal with the right stimuli. In one instance, this meant that the manufacturer would "not always need users, (they) can do a heuristic analysis prior to involving users to gain insights in possible design changes" (Company 7).

Scheduling time with users was described as "difficult and frustrating" (Company 14). Company 9 communicated that the cadence of medical device development progress did not necessarily match the clinical calendar. Further, that "Hospitals and physicians were slow to respond and then it takes forever to explain our (industry) goals and be approved to interact." Once approved, challenges of scheduling were still problematic with regards to matching the needs of device development.

Once a meeting was scheduled further challenges existed, such as what questions to ask and how to analyze the information. This is evident in the quote by Company 3 , "it was difficult knowing when and what to listen to in order to translate the information into design criteria...to know we've asked the right questions to the right people as we (industry) rarely have the opportunity to validate the information (with users)" (Company 3).

Seven companies reported that the attitude and ego of the user could also be a hindrance to collaboration. Each of these companies had physicians as users and only one of the companies (Company 17) had in-house industrial design. The personalities of the difficult users were described as "not conducive to collaboration as they believe they sit at the right hand of god" (Company 16). As a result, the management of social dynamics became increasingly important so as to not offend users and thereby hinder the collaborative process. In addition, companies described a reluctance to change and the slow adoption of new technologies within the practice of medicine. Company 13 approached this problem with iterative changes rather than radical disruptions.

Device industry development teams were concerned with the ownership of intellectual property and, as a result, rarely undertook brainstorm activity with users. Company 11 commented that users struggled to think of alternative means of clinical 
practice and that, "they (users) were not good at it (brainstorming) and have a difficult time thinking of alternative ways of doing their job" (Company 11). Several industry participants described users as having a "lack of mechanical aptitude" and a requirement to "design for the least intelligent person" (Company 4, 11, 14).

Six Companies reported that the conflicting opinions of users were a challenge for design. Typically, "the marketing group suggests, the more (users) the merrier in opinion, however different opinions that contradict each other become difficult to decipher in device design" (Company 12). Selecting the right number of users becomes a challenge for the device industry as "users may reveal something that was never a requirement and then the design needs to change to accommodate desires. This was after we've started our design control process" (Company 12).

There was a cost associated with involving users and the "activity was not cheap" (Company 18). Sometimes the compensation went into education funds so as to avoid official reporting. In other instances, an official honorarium was required and expected. It was apparent that users did not review or interact with industry professionals without expectation of reward. The financial burden did "impact the ability to gather a wide opinion and making decisions on limited information, can make for bad decisions" (Company 12). In order to contain costs, the overall number of study participants, their backgrounds, corporate user compensation norms and the number of study locations were explored and balanced.

The management and requirements of healthcare laws $(\mathrm{N}=5)$ and IRB $(\mathrm{N}=2)$ reporting were viewed as a challenge for companies to engage the user. Healthcare laws, such as the Sunshine Act in the US, require the reporting of any compensation for physicians that resulted in physician hesitancy due to conflict of interest. To avoid conflict of interest issues, users were often engaged in "preceptorship roles wherein they were paid to train product development personnel in the procedure during observations" (Company 2). This enabled the device community to observe but always required a formal agreement, documentation and compensation. Company 18 commented that if additional approval such as IRB review was necessary, an additional three months might be required in order to run a device study. As a result, this situation was avoided. IRB requirements were viewed as burdensome for 
smaller organizations with limited budgets and tight timelines. There was confusion amongst the study participants in regards to the need for IRB oversight for human factors studies. This was determined by the individual study requirements e.g. direct patient interaction always required IRB approval. The submission and management of IRB approval was placed on the usability study team with support from the product development team. Additional budget in time and money was required for studies incorporating IRB approval.

\section{Discussion}

As the literature review highlights, the application of human factors standards within the medical device design process is an expectation according to both the FDA and MHRA. (FDA 2016; MHRA 2016). Barriers in communication regarding human factors processes have been identified (Vincent et al. 2014) and the user was defined to vary dependent upon the device and can include a provider, patient, parent or caregiver (Money et al. 2011). The literature provides background on specific human factors process requirements however does not include analysis in the implementation of these processes.

Using a multiple case study design, this research enabled depth into the processes and attitudes in meeting the human factors standards as well as overall collaboration with users. The participants selected were those responsible for the overall design process, direction and activities of the design team. Their acknowledgement of human factors standards demonstrates the effectiveness of agency requirements.

This paper has explored current practices within the medical device industry for the purposes of improving device design; applying human factors principles, the influences of regulatory bodies and interaction with users. The results confirm that all medical device development activities begin with both the clinical problem and interactions with the user. This verifies the impact of regulatory standards to promote user-centered design and that interaction was welcomed but difficult for medical device companies to implement. Through agency mandates, there were specific activities and methods that promoted user involvement. However, based on the perceptions in the industry, users were not always as helpful nor were they necessarily easy to work with. 
This research indicates a total of four specific issues between device users and industry in regards to the application of human factors standards. This includes the following:

1. Recognition of agency mandates required involvement of the user but access to users remained difficult.

2. Users drove the approach to design but were perceived to lack understanding of their impact and the development process.

3. Device evaluations, workshops/labs were activities promoting user involvement and were conducted under formal circumstances, with contract formalities that met a requirement rather than a casual exchange with frequent exchanges.

4. Users expected compensation to collaborate with the device industry and their attitudes could negatively impact the application of human factors processes.

\section{Conclusion}

The barriers presented in this research have the potential to be resolved but only with greater commitment by both users and device developers. As a result, alternative practices that may further promote collaborative device development can be recommended. These include the following:

- To enable access to users and connecting interested users with device developers, targeted social media may serve as a direct connection.

- To ensure that users are familiar with the latest practices, developers should consider rotating device users (physicians/nurses/techs) as device development team members with limited time away from practice.

- To more effectively collaborate with users, educational programs could be produced to acquaint users with the product development practice.

- To ensure users understand their impact on device development, the contribution and limitations of user input could be more clearly defined the beginning of all activities related to device development. 
- To ensure consistent user input throughout the development process, a tool or means of verifying user criteria continuously in the design process beyond formal testing of prototypes could be employed.

- To identify the most appropriate user for collaboration, a system could be developed as a means of filtering/directing users with particular personality traits that would be most effective at various stages in device development.

The application of human factors standards and the practice of user-centered design remain a priority in the industry for the development of safe, effective and usable devices. Despite, challenges, it is clear that the industry respects these regulatory requirements and values the input of device users.

\section{Acknowledgements}

This research was the result of collaborations between Loughborough University Design School and the University of Cincinnati's Medical Device Innovation \& Entrepreneurship Program. It would not have been possible without the cooperation and volunteer time of those medical device manufacturers included in this research. We are grateful and appreciative of their insights and willingness to participate.

This research did not receive any specific grant from funding agencies in the public, commercial or not-for-profit sectors.

\section{References}

Association for the Advancement of Medical Instrumentation (AAMI, 2013. A roundtable discussion: understanding medical devices and users in context. Biomed Instrum Technol., Fall, pp.8-13.

de Ana, F.J. et al., 2013. Value driven innovation in medical device design: a process for balancing stakeholder voices. Annals of biomedical engineering, 41, pp.1811-21. Available at: http://www.ncbi.nlm.nih.gov/pubmed/23483372.

Battarbee, K. \& Koshinen, I., 2005. Co-Experience: User experience as interaction. CoDesign; International Journal of CoCreation in Design and the Arts, 1(1), p.18.

Bauer, M., 1996. The narrative interview: Comments on a technique for qualitative data collection, Available at: http://opensigle.inist.fr/handle/10068/537304.

Binder, T., Brandt, E. \& Gregory, J., 2008. Editorial, Design Participation(-s). CoDesign; International Journal of CoCreation in Design and the Arts, 4(1), pp.1-3.

Bussolon, S., 2009. Card sorting, category validity, and contextual navigation. Journal of Information Architecture, 1(2), pp.5-29. Available at: http://journalofia.org/volume1/issue2/02-bussolon/jofia-0102-02-bussolon.pdf.

Cafazzo, J.A. \& St-Cyr, O., 2012. From discovery to design: the evolution of human factors in healthcare. Healthcare quarterly (Toronto, Ont.), 15 Spec No, pp.24-29.

Crisp, S., 1996. The medical device directives and their impact on the development and manufacturing of medical implants. Proceedings of the Institution of Mechanical 
Engineers. Part $H$, Journal of engineering in medicine, 210(4), pp.233-239.

Elf, M. et al., 2007. Using system dynamics for collaborative design: a case study. BMC Health Services Research, 7(1), pp.1-12. Available at: http://dx.doi.org/10.1186/1472-6963-7-123.

Fairbanks, R.J. \& Wears, R.L., 2008. Hazards with medical devices: the role of design. Annals of emergency medicine, 52, pp.519-521.

FDA, 2011. Applying Human Factors and Usability Engineering to Optimize Medical Device Design,

FDA CDRH, 1997. Guidance Documents (Medical Devices and Radiation-Emitting Products) - Design Control Guidance For Medical Device Manufacturers, USA: Center for Devices and Radiological Health. Available at: http://www.fda.gov/medicaldevices/deviceregulationandguidance/guidancedocumen ts/ucm070627.htm [Accessed February 1, 2015].

Gilman, B.L., Brewer, J.E. \& Kroll, M.W., 2009. Medical device design process. Conference proceedings: ... Annual International Conference of the IEEE Engineering in Medicine and Biology Society. IEEE Engineering in Medicine and Biology Society. Conference, 2009, pp.5609-5612.

Grocott, P., Weir, H. \& Ram, M.B., 2007. A model of user engagement in medical device development. International journal of health care quality assurance, 20(6), pp.484493.

Justiniano, J. \& Gopalaswamy, V., 2003. Practical Design Control implementation for Medical Devices, Boca Raton, Florida 33431: Interpharm CRC.

Kvale, S. \& Brinkmann, S., 2009. InterViews: learning the craft of qualitative research interviewing, Los Angeles: Sage Publications.

Lin, L., Vicente, K.J. \& Doyle, D.J., 2001. Patient safety, potential adverse drug events, and medical device design: a human factors engineering approach. Journal of Biomedical Informatics, 34(4), pp.274-284.

Martin, J.L. \& Barnett, J., 2012. Integrating the results of user research into medical device development: insights from a case study. BMC medical informatics and decision making, 12, p.74.

Medina, L.A., Kremer, G.E.O. \& Wysk, R.A., 2012. Supporting medical device development: a standard product design process model. Journal of Engineering Design, pp.1-37.

MHRA, 2016. Human Factors and Usability Engineering - Guidance for Medical Devices Including Drug-device Combination Products, Available at: https://www.gov.uk/government/uploads/system/uploads/attachment_data/file/5284 95/MHRA_Human_factors_draft_guidance_June_2016.pdf

Miles, M.B., Huberman, A.M. \& Saldana, J., 2014. Qualitative Data Analysis, United States of America: Sage.

Miles, M.B., Huberman, A.M. \& Saldaña, J., 2013. Qualitative Data Analysis: A Methods Sourcebook, SAGE Publications. Available at: https://books.google.com/books?id=3CNrUbTu6CsC\&pgis=1 [Accessed March 1, 2015].

Money, A.G. et al., 2011. The role of the user within the medical device design and development process: medical device manufacturers' perspectives. BMC medical informatics and decision making, 11, p.15.

Ogrodnick, P., 2013. Medical Device Design: Innovation from Concept to Market, London: Elsevier Inc.

Ogrodnik, P., 2013. Medical Device Design: Innovation from Concept to Market, London: Elsevier.

Panescu, D., 2009. Medical device development. In Conference proceedings : Annual 
International Conference of the IEEE Engineering in Medicine and Biology Society. IEEE Engineering in Medicine and Biology Society. Annual Conference. pp. 5591-4. Rugg, G. \& McGeorge, P., 2005. The sorting techniques: A tutorial paper on card sorts, picture sorts and item sorts. Expert Systems, 22(3), pp.94-107.

Sanders, E.B.-N., 2002. From user-centered to participatory design approaches. Design and the social sciences: Making connections, pp.1-8.

Taylor, P., Furniss, D. \& Blandford, A., 2007. Understanding emergency medical dispatch in terms of distributed cognition : a case study Understanding emergency medical dispatch in terms of distributed cognition : a case study. , (July 2014), pp.37-41.

Vincent, C.J., Li, Y. \& Blandford, A., 2014. Integration of human factors and ergonomics during medical device design and development: It's all about communication. Applied Ergonomics, 45(3), pp.413-419. Available at: http://www.sciencedirect.com/science/article/pii/S0003687013001208.

Whitmore, E., 2004. Development of FDA-Regulated Medical Devices Prescription Drugs, Biologics and Medical Devices, Milwaukee, Wisconsin: American Society for Quality, Quality Press.

Wiklund, M.E. \& Wilcox, S.B., 2005. Designing usability into medical products, Boca Raton, Fla: CRC Press. Available at: http://uc.summon.serialssolutions.com/link/0/eLvHCXMwY2BQMEg2TwLWuqZpFm aWackmwArSKBmo0DQFWPUmGhqnolzlg1SauwkxMKXmiTJlu7mGOHvolibHQ4c w4pOMzcEzYhWGYgy8iaCl33kl4C1iKelMrGnAeEoVB5Wd4kBzxBk4liyNgvwiLAMg XCEYV68Yv19Jr7BEHFhUg6NZ11DPFABvESsG.

Yin, R.K., 2013. Case Study Research: Design and Methods 5th ed., SAGE Publications. Available at: https://books.google.com/books?id=OgyqBAAAQBAJ\&pgis=1 [Accessed March 1, 2015].

Zenios, S., Makower, J. \& Yock, P., 2010. Biodesign: the process of innovating medical technologies, Cambridge University Press. 